\title{
Alteration of epithelial-mesenchymal transition markers in human normal ovaries and neoplastic ovarian cancers
}

\author{
BO-RIM YI $^{{ }^{*}}$, TAE-HEE KIM ${ }^{2 *}$, YE-SEUL KIM ${ }^{1}$ and KYUNG-CHUL CHOI ${ }^{1}$ \\ ${ }^{1}$ Laboratory of Biochemistry and Immunology, College of Veterinary Medicine, Chungbuk National University, \\ Cheongju, Chungbuk; ${ }^{2}$ Department of Obstetrics and Gynecology, College of Medicine, \\ Soonchunhyang University, Bucheon, Republic of Korea
}

Received August 4, 2014; Accepted September 19, 2014

DOI: 10.3892/ijo.2014.2695

\begin{abstract}
Most ovarian cancers originate in the ovarian surface epithelium (OSE). Ovarian cancers might undergo epithelial-mesenchymal transition (EMT) in response to various mediators or regulators such as EMT-inducing factors. In this study, ovarian tumor specimens from patients were analyzed to demonstrate alteration of EMT-related markers according to benign and malignant types of ovarian cancers. In the three ovarian cancer cell lines, OVCAR-3, SKOV-3, and BG-1, the expression of epithelial (E-cadherin) and mesenchymal (vimentin) cell markers was identified by RNA and protein analysis. OVCAR-3 and BG-1 cells strongly expressed E-cadherin as well as morphological features such as epithelial cells, but vimentin was not observed. In contrast to these cancer cells, SKOV-3 showed a typical phenotype of mesenchymal cells. Alteration of EMT markers and EMT-related transcriptional factors were confirmed in clinical ovarian tissue samples obtained from 74 patients. E-cadherin was expressed in $57.1 \%$ of benign tumors, while vimentin was expressed in $83.3 \%$ of normal ovaries by western blot analysis in the tissue specimens. Evaluation of the EMT-associated transcriptional factors Snail, Slug, and Twist revealed that Snail was overexpressed by 7.1-fold in malignant ovarian cancer compared to normal ovaries or benign tumors. Although expression levels of other factors were higher in benign and malignant ovarian tumors, they were not closely correlated with the aforementioned ovarian cancer types. Overall, Snail may affect the EMT process in ovarian cancer development and upregulation of Snail expression followed by the downregulation of E-cadherin enhances the invasiveness of ovarian cancer.
\end{abstract}

Correspondence to: Dr Kyung-ChulChoi,Laboratory of Biochemistry and Immunology, College of Veterinary Medicine, Chungbuk National University, Naesudong-ro 52, Cheongju, Chungbuk 361-763, Republic of Korea

E-mail:kchoi@cbu.ac.kr

"Contributed equally

Key words: ovarian cancer, EMT, E-cadherin, vimentin, Slug, Snail

\section{Introduction}

Epithelial-mesenchymal transition (EMT) is a critical process in cell differentiation, morphogenesis, growth and change of function (1). EMT is a cellular mechanism recognized as a central feature of normal physiological development such as organogenesis during embryonic development and wound healing (2). Several developmental milestones, including gastrulation, neural crest formation, and heart morphogenesis, rely on the plastic transition between epithelial cells and mesenchymal cells (3). However, dysregulated EMT appears to occur in cancer progression and metastasis, as well as the pathogenesis of chronic degenerative fibrotic disorders in several organs (4). Epithelial and mesenchymal cells differ in function and characteristics. Epithelial cells are closely adjoined by specialized membrane structures such as desmosomes, as well as tight, adherent, and gap junctions, and have apical-basolateral polarization (5). Conversely, mesenchymal cells do not form an organized cell layer, nor do they have the same apical-basolateral organization, polarization of the cell surface molecules (6). In culture, epithelial cells grow in clusters, whereas mesenchymal cells have a spindle shaped and fibroblast-like morphology (7). During the EMT process, epithelial cells are removed of cellular polarity and epithelial cell-cell as well as cell-matrix adhesion contacts are remodeled (8). Epithelial cells also acquire fibroblastlike properties and show reduced intercellular adhesion and increased motility (9). EMT is characterized by increased expression of mesenchymal markers (vimentin, thrombospondin, N-cadherin, vitronectin), increased expression of extracellular matrix compounds (fibronectin), decreased expression of epithelial markers (E-cadheirn, collagen IV, occludin, desmoplakin, and mucin 1), altered location of transcription factors ( $\beta$-catenin, Snail, Slug, Twist, sox 10, and $\mathrm{NF}-\kappa \mathrm{B})$ and activation of kinase (Erk1/2 and PI3K/Akt) (10).

A critical molecular feature of EMT is downregulation of E-cadherin, a cell adhesion molecule that acts as a calcium-dependent transmembrane glycoprotein in the plasma membrane of most normal epithelial cells in embryonic and adult tissues (11). Two main types of consensus binding sites have been shown to downregulate E-cadherin expression, Ets sites and palindromic E-box (E-pal) (3). E-boxes are characterized by the consensus sequence, 5'-CACCTG-3', and 
consist of three E-boxes in the human E-cadherin promoter: two upstream from the transcription start site and one in exon 1 (12). Transcriptional repression of E-cadherin has several important consequences that are of direct relevance to the EMT process $(13,14)$. Several transcriptional factors that repress transcription of E-cadherin have been identified during development and translocation, including Snail, Slug, Twist, and ZEB1/2 downregulation, which induces EMT (15). In particular, the zinc finger transcriptional repressors Snail and Slug have been implicated in repression of E-cadherin transcription in vitro (16), as well as in mesoderm formation together with Twist (17).

Ovarian cancer is the leading cause of death from gynecological tumors, and epithelial ovarian cancers originating from the ovarian surface epithelium (OSE) constitute $\sim 90 \%$ of human ovarian neoplasms (18). Unfortunately, this disease is often not diagnosed until in advanced stages and not before metastatic setting of the tumor due to lack of early stage detection (19). OSE covering a nonovulating ovary is a stationary mesothelium that exhibits epithelial and mesenchymal characteristics with the capacity to give rise to inclusion cysts through loss of mesenchymal characteristics (20). Mesodermally derived normal OSE shows epithelial and mesenchymal features characterized by the expression of both keratin and vimentin, and OSE cells can be converted to a mesenchymal, fibroblast-like phenotype, at least in vitro (21). Interestingly, only low levels of E-cadherin are prominent in OSE cells, while E-cadherin is present in OSE cells covering deep clefts, inclusion cysts and ovarian tumors (22). Repression of E-cadherin has been described in a high percentage of borderline ovarian tumors and carcinomas when compared with benign tumors. Moreover, ascites cells with low E-cadherin expression were found to be more invasive than solid tumor cells (23). This initial shift towards a more differentiated phenotype early in tumor progression appears to be followed by reacquisition of mesenchymal features in more advanced ovarian tumors, involving a secondary loss of E-cadherin (24). Therefore, ovarian carcinomas show unique features when compared to other epithelial derived cancers.

We focused on EMT marker expression among ovarian types. We report that E-cadherin was highly expressed in benign tumors when compared to normal ovaries or malignant tumors. As a result, expression of EMT-associated marker is dependent on ovarian status, and is related to transcription factor via regulation of the expression of E-cadherin. These results support the hypothesis that detection of EMT-related marker alteration in OSE cells may be important in the prognosis and diagnosis of ovarian neoplasms and thus provide protection from the initiation of neoplastic transformation.

\section{Materials and methods}

Clinical human ovarian cancer sample. Clinical specimens of human normal ovaries and ovarian cancers were provided by Dr T.H. Kim at the Department of Obstetrics and Gynecology (Soonchunhyang University, Bucheon, Kyeonggido, Korea) between 2011 and 2013. Our ovarian materials consist of 44 normal ovarian samples, 15 benign and 3 borderline ovarian tumors and 12 malignant tumor samples as shown in Table I. Ovarian tissue samples from a total of 74 patients
Table I. Classification of normal ovaries and ovarian cancers.

Number

\begin{tabular}{lc}
\hline Type & \\
Normal & 44 \\
Benign & 15 \\
Borderline & 3 \\
Malignant & 12 \\
Total & 74 \\
Ages & \\
$\leq 45$ & 35 \\
$>46$ & 39 \\
Weight of mass (ave.) & \\
Normal & - \\
Benign & $179.73 \pm 68.55$ \\
Borderline & $826.50 \pm 327.39$ \\
Malignant & $272.63 \pm 246.23$ \\
\hline
\end{tabular}

who had undergone primary surgery for newly diagnosed advanced stage of ovarian abnormalities were included in this study. Histological type and grade were evaluated according to the World Health Organization (WHO) by an experienced pathologist at Soonchunhyang University Hospital. The specimens were divided into three pieces and then stored at $-80^{\circ} \mathrm{C}$ until extraction of RNA and protein samples or fixed in $10 \%$ normal formalin for histopathological analysis. Most ovarian tissues were obtained by cystectomy, salpingooophorectomy, and salpingectomy. Additionally, only patients who had not undergone chemotherapy or radiotherapy prior to surgery were included in the study.

Cell culture. Three ovarian cancer cell lines of human origin were used, SKOV-3, OVCAR-3, and BG-1 cells. The ovarian adenocarcinoma cell lines, SKOV-3 and OVCAR-3, were purchased from the Korean Cell Line Bank (KCLB, Seoul, Korea). An adenocarcinoma cell line, BG-1, was provided by Dr K.S. Korach (National Institute of Environmental Health Sciences, Research Triangle Park, NC, USA). SKOV-3 and BG-1 cells were routinely cultured in Dulbecco's modified Eagle's medium (DMEM; Hyclone Laboratories, Inc., Logan, UT, USA) supplemented with $10 \%$ (v/v) heat inactivated fetal bovine serum (FBS; Hyclone Laboratory Inc.), antibiotics (100 U/ml penicillin and $100 \mu \mathrm{g} / \mathrm{ml}$ streptomycin; Cellgro Mediatech Inc., Manassas, VA, USA), and $10 \mathrm{mM}$ HEPES (Gibco, Carlsbad, CA, USA) in a humidified incubator at $37^{\circ} \mathrm{C}$ under $5 \% \mathrm{CO}_{2}$. OVCAR-3 cells were grown in RPMI-1640 medium containing $15 \% \mathrm{FBS}$ and maintained in a humidified atmosphere of $5 \% \mathrm{CO}_{2}$ at $37^{\circ} \mathrm{C}$. Cells were trypsinized with trypsin/EDTA and medium was changed twice a week.

Total RNA extraction and polymerase chain reaction (PCR). Total RNA of three ovarian cancer cells and clinical samples was extracted using RNA extraction solution and TRIzol reagent (Invitrogen Life Technologies, San Diego, CA, USA) according to the general protocols. The complementary DNA (cDNA) was obtained from $1 \mu \mathrm{g}$ of total RNA using murine 
Table II. Sequences of reverse-transcription or real-time PCR primer pairs.

\begin{tabular}{|c|c|}
\hline mRNA & Sequence $\left(5^{\prime}-3^{\prime}\right)$ \\
\hline E-cadherin & $\begin{array}{ll}\mathrm{F} & \text { TCCCATCAGCTGCCCAGAAA } \\
\mathrm{R} & \text { ATTGTCCTTGTGTCCTCAGT }\end{array}$ \\
\hline Vimentin & $\begin{array}{ll}\mathrm{F} & \text { ACGCCATCAACACCGAGTTCA } \\
\mathrm{R} & \text { AACTGTTACGCAGAGCCAGTG }\end{array}$ \\
\hline Snail & $\begin{array}{ll}\text { F } & \text { AAGCTTCCATGGCGCGCTCTTTCCTCGTCAGGAAGCCC } \\
\text { R } & \text { GGATCCTCAGCGGGGACATCCTGAGCAGCCGGACTCTTG }\end{array}$ \\
\hline Slug & $\begin{array}{ll}\mathrm{F} & \text { CCTTCCTGGTCAAGAAGCAT } \\
\mathrm{R} & \text { CACAGTGATGGGGCTGTATG }\end{array}$ \\
\hline Twist & $\begin{array}{ll}\mathrm{F} & \text { GGATCCATGATGCAGGACGTGTCCAGCTCGCCA } \\
\mathrm{R} & \mathrm{CTCGAGCTAGTGGGACGCGGACATGGACCAGGC}\end{array}$ \\
\hline GAPDH & $\begin{array}{ll}\text { F } & \text { ATGTTCGTCATGGGTGTGAACCA } \\
\mathrm{R} & \text { TGGCAGGTTTTTCTAGACGGCAG }\end{array}$ \\
\hline
\end{tabular}

leukemia virus reverse transcriptase (MMLV-RT; iNtRON Biotechnology, Sungnam, Kyeonggido, Korea), 10 pM dNTP (Bioneer, Daejeon, Korea), random nonamer primer (Takara Bio., Shiga, Japan), 5X RT buffer (iNtRON Biotechnology) and RNase inhibitor (iNtRON Biotechnology) for $1 \mathrm{~h}$ at $37^{\circ} \mathrm{C}$. Prepared cDNA was amplified using 2.5 U of Taq polymerase (iNtRON Biotechnology), 10X PCR buffer (iNtRON Biotechnology), $10 \mathrm{pM}$ of dNTP (Bioneer), and sense/antisense primers for E-cadherin, vimentin, and glyceraldehyde-3-phosphate dehydrogenase (GAPDH) genes. Each primer is listed in Table II. The PCR reaction consisted of 30 cycles of denaturation at $95^{\circ} \mathrm{C}$ for $30 \mathrm{sec}$, annealing at $58^{\circ} \mathrm{C}$ for $30 \mathrm{sec}$ and extension at $72^{\circ} \mathrm{C}$ for $30 \mathrm{sec}$. Amplified products were separated on $1.5 \%$ agarose gel containing ethidium bromide $(\mathrm{EtBr}$; Sigma-Aldrich Co., St. Louis, MO, USA) and confirmed using a Gel Doc 2000 (Bio-Rad Laboratories Inc., Hercules, CA, USA).

Real-time PCR. The real-time PCR mixture was composed of 2X SYBR green premix (Takara Bio.), ROX (Takara Bio.) as a reference dye, and reverse and forward primers (Bioneer) specific for the EMT-related transcriptional factor gene. The reaction consisted of denaturation at $95^{\circ} \mathrm{C}$ for $15 \mathrm{sec}$, followed by annealing at $58^{\circ} \mathrm{C}$ for $20 \mathrm{sec}$ and extension at $72^{\circ} \mathrm{C}$ for $15 \mathrm{sec}$ (40 cycles). The real-time PCR reaction was carried out in triplicate for each sample. GAPDH was used for normalization and the mRNA levels of Snail, Slug, and Twist genes were compared. The mRNA levels of these genes were determined using the $2^{-\Delta \Delta \mathrm{Ct}}$ method and the specific primer pairs listed in Table II.

Protein extraction and quantification. To analyze the levels of EMT related protein, whole lysates were extracted from ovarian cancer cell lines and clinical ovary samples. First, 1X RIPA solution (50 mM Tris-HCl, pH 8.0, 150 mM NaCl, 1\% NP-40, $0.5 \%$ deoxycholic acid, and $0.1 \%$ sodium dodecyl sulfate) was applied to the three ovarian cancer cell lines cultured in dishes to obtain the protein. Next, part of the excised normal ovaries or ovarian cancers from patients was used to prepare the whole tissue lysate. Briefly, clinical ovarian samples were homogenized in PRO-PREP ${ }^{\circledR}$ lysis buffer (iNtRON Biotechnology). After overnight incubation at $4^{\circ} \mathrm{C}$, samples were centrifuged at $14,000 \mathrm{rpm}$ for $30 \mathrm{~min}$ at $4^{\circ} \mathrm{C}$. The concentration of total protein of ovarian cancer cells and clinical samples was then determined using bicinchoninic acid (BCA; Sigma-Aldrich Co.) and copper (II) sulfate (Sigma-Aldrich Co.) mixture. Next, $150 \mu \mathrm{l}$ of the solution was mixed with total protein and incubated at $37^{\circ} \mathrm{C}$. After $30 \mathrm{~min}$, the absorbance was measured at $640 \mathrm{~nm}$. Subsequently, $40 \mu \mathrm{g}$ of proteins were mixed with protein loading buffer (Bio-Rad Laboratories Inc.) containing $\beta$-mercaptoethanol and heated at $100^{\circ} \mathrm{C}$ for $5 \mathrm{~min}$.

Western blot analysis. Whole cell lysates were resolved by $12 \%$ SDS-polyacrylamide gel electrophoresis (SDS-PAGE), after which fractionated proteins were transferred to polyvinylidene difluoride (PVDF) transfer membrane (Bio-Rad Laboratories Inc.). The membrane was then blocked with $5 \%$ skim milk (Bio-Rad Laboratories Inc.) for $2 \mathrm{~h}$ to inhibit non-specific interaction with primary antibody, then washed four times in 1X TBS buffer (adjusted to $\mathrm{pH} 7.6$ using $\mathrm{HCl}$ ) containing $0.1 \%$ (v/v) Tween-20 (Bio-Rad Laboratories Inc.). Membrane was incubated with primary antibody, mouse monoclonal anti-E-cadherin (1:1,000 dilution, Abcam plc., Cambridge, UK), anti-vimentin (1:1,000 dilution, Abcam plc.), or anti-GAPDH (1:1,000 dilution, Santa Cruz Biotechnology, CA, USA) in $1 X$ TBS with $3 \%$ (w/v) BSA (Sigma-Aldrich Co.) and $0.1 \%$ (v/v) Tween-20 overnight at $4^{\circ} \mathrm{C}$. The next day, samples were incubated with specific antibody followed by the appropriate horseradish peroxidase (HRP) conjugated secondary antibody, goat anti-mouse IgG (1:3,000 dilution, Bio-Rad Laboratories Inc.), for $2 \mathrm{~h}$. Positive immunoreactive proteins were detected using an ECL kit (West-Q Chemiluminescent Substrate Plus kit, GenDEPOT, Barker, TX, USA).

Hematoxylin and eosin (H\&E) staining. Clinical human normal ovaries and ovarian cancer tissue were fixed in $10 \%$ normal 
formalin (Sigma-Aldrich Co.), embedded in paraffin blocks and cut with a sliding microtome (3- $\mu \mathrm{m}$ sections). After deparaffination and rehydration with xylene and ethanol, the slides were stained using hematoxylin and eosin (Sigma-Aldrich Co.). To prevent sample contamination, stained slides were hydrated and mounted using a hydrophobic mounting solution. The morphology of tissues derived from ovaries was observed by light microscopy using a BX51 microscope (Olympus, Japan).

Immunohistochemistry (IHC). For histological analysis, paraffin-embedded sections were deparaffinized and rehydrated by xylene, ethanol, and tap water. Antigen retrieval was accomplished by boiling the slides in a microwave for $10 \mathrm{~min}$ in a chamber containing $0.01 \mathrm{M}$ citrate buffer ( $\mathrm{pH}$ 6.0). To quench the endogenous peroxidase, tissue slides were placed in $0.3 \%$ methanol/hydrogen peroxidase (Sigma-Aldrich Co.) for $30 \mathrm{~min}$. To block the non-specific binding of primary antibodies, slides were exposed to $10 \%$ normal goat serum (Vector Laboratories, Burlingame, CA, USA) for $1 \mathrm{~h}$. Subsequently, the slides were incubated with a mixture of anti-E-cadherin (1:100, Abcam plc.) or anti-vimentin (1:100, Abcam plc) as the primary antibody in $5 \%$ bovine serum albumin (Sigma-Aldrich Co.) in a humidified chamber at $4^{\circ} \mathrm{C}$ overnight. The next day, the slides were washed three times in 1X PBS-T $(\mathrm{pH}$ 7.4) and then incubated with appropriate biotinylated secondary antibodies (1:500 dilution, Vector Laboratories) for $30 \mathrm{~min}$ at room temperature. Slides were subsequently rinsed with 1X PBS-T for $10 \mathrm{~min}$, after which Vectastain Universal Elite ABC kit reagent (Vector Laboratories) was applied for $30 \mathrm{~min}$. Immunoreactive complexes were detected using DAB substrate (Sigma-Aldrich Co.) and counter stained with hematoxylin. Finally, slides were mounted with a cover slip using mounting solution. All slides were visualized under a BX51 light microscope for digital photography.

Statistical analysis. Statistical analyses were performed using GraphPad Prism 5 (GraphPad Software, San Diego, CA, USA). Statistical differences in RNA expression levels were identified by one-way ANOVA followed by Tukey's test. Results were expressed as the mean \pm SD. Provability values $<0.05$ were considered statistically significantly.

\section{Results}

Expression of EMT related markers in ovarian cancer cells. To observe the fate of EMT related markers in the three ovarian cancer cell lines, we conducted reverse-transcription PCR and western blot analysis to detect E-cadherin and vimentin without any treatment. Reverse-transcription PCR showed that E-cadherin expression was significantly higher in OVCAR-3 and BG-1 ovarian cancer cells, while its expression was absent in SKOV-3 cells (Fig. 1A). Additionally, the expression levels of vimentin RNA as a typical mesenchymal cell marker were observed in SKOV-3 and OVCAR-3 ovarian cancer cells. In addition to RNA analysis, E-cadherin protein was expressed in OVCAR-3 and BG-1 cells, but vimentin was only expressed in SKOV-3 cells (Fig. 1B). Of the three ovarian cancer cell lines assayed, two cells showed a lack of vimentin and the presence of E-cadherin, which is a typical pattern for EMT related markers. These two cells lines (OVCAR-3 and BG-1) have a

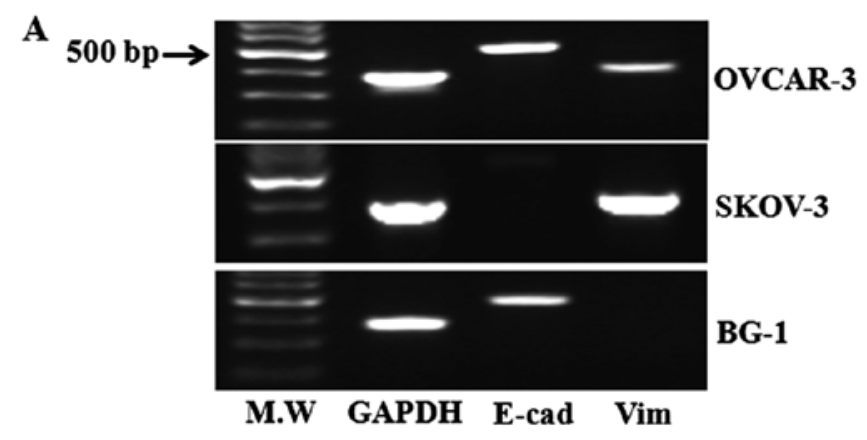

B

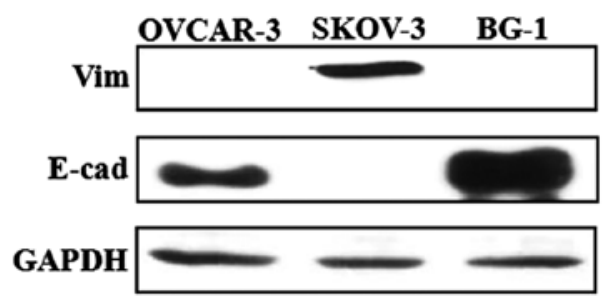

Figure 1. RNA and protein levels of mesenchymal and epithelial cell markers in three ovarian cancer cells lines, BG-1, SKOV-3, and OVCAR-3. (A) RNA levels of mesenchymal and epithelial cell markers in the three ovarian cancer cell lines. Total RNA was extracted by RNA extraction solution in ovarian cancer cell solution and cDNA was obtained from $1 \mu \mathrm{g}$ of RNA. The cDNA was amplified by PCR and confirmed by electrophoresis in agarose gel containing EtBr. (B) Protein levels of EMT related marker in ovarian cancer cell lines. Total protein was extracted by 1 X RIPA solution to confirm the expression of E-cadherin and vimentin protein in ovarian cancer cells. Total protein was separated by SDS-PAGE and transferred to PVDF membrane. Next, samples were treated with primary antibody, anti-E-cadherin $(1: 1,000$ dilution) and anti-vimentin (1:1,000 dilution) overnight, then incubated with the secondary anti-mouse antibody (1:3,000 dilution) for $2 \mathrm{~h}$.

cuboidal, epithelial morphology, while SKOV-3 cells have a spindle-shaped morphology (data not shown).

E-cadherin/vimentin expression in clinical normal ovary and ovarian cancers. To measure the expression levels of E-cadherin RNA in clinical ovarian cancers and normal ovary samples, we randomly selected four groups: 1, six normal ovaries; 2 , seven benign ovarian cancers; 3 , three malignant ovarian cancers; and 4, two borderline ovarian cancers. Upon analysis of E-cadherin RNA expression, E-cadherin was expressed in $50 \%$ of normal ovaries and $66.6 \%$ of malignant ovarian cancers, whereas it was expressed in $85.7 \%$ of benign ovarian cancers (Fig. 2A).

We also conducted western blot analysis to measure protein levels of vimentin and E-cadherin in human clinical samples obtained from patients. E-cadherin was observed in 16.7, $57.1,50$ and $16.7 \%$ of normal ovaries, benign, borderline, and malignant ovarian tumors, respectively (Fig. 2B). Conversely, alteration of vimentin protein was observed in 83.3, 57.1, 50 and $66.7 \%$ of normal ovaries, benign, borderline, and malignant tumors respectively. Therefore, the E-cadherin gene was upregulated in benign ovarian tumors compared to normal ovary tissues and decreased in malignant ovarian tumors.

Quantification of EMT-related factors in the ovarian tissue samples. To identify differences in the expression of EMT-associated transcription factor between normal ovaries and ovarian cancers, we conducted additional analyses 


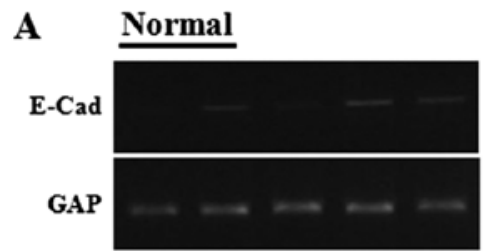

B
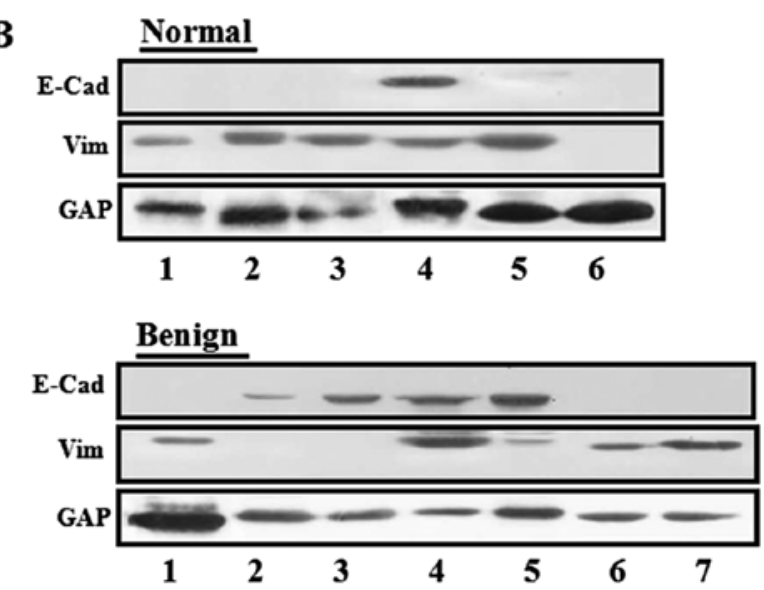
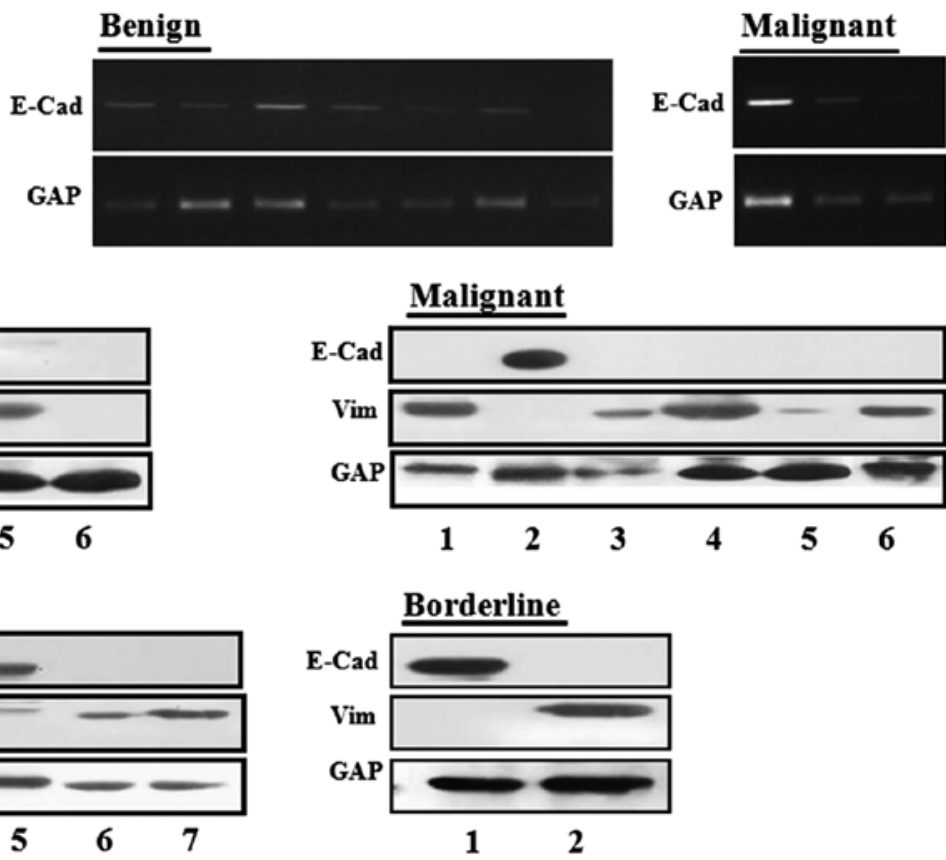

Figure 2. Alteration of E-cadherin and vimentin RNA and protein in human normal ovaries and ovarian cancer samples provided by patients. (A) Expression of E-cadherin gene in human samples. To analyze the expression of E-cadherin RNA, total RNA was obtained from human clinical samples from three groups, normal ovary, benign, and malignant ovarian tumor. cDNA was synthesized by MMLV reverse transcriptase and PCR product was separated by agarose gel electrophoresis after PCR. (B) Expression of E-cadherin and vimentin protein in human ovarian samples. Total protein was extracted using 1X RIPA protein extraction solution and SDS-PAGE was conducted to separate samples based on the molecular size of the proteins. Separated proteins were transferred from the polyacrylamide gel to the PVDF membrane. Next, the membrane was incubated with primary antibody, anti-E-cadherin (1:1,000 dilution) and antivimentin (1:1,000 dilution) overnight and HRP-conjugated secondary anti-mouse antibody (1:3,000 dilution) for $2 \mathrm{~h}$.

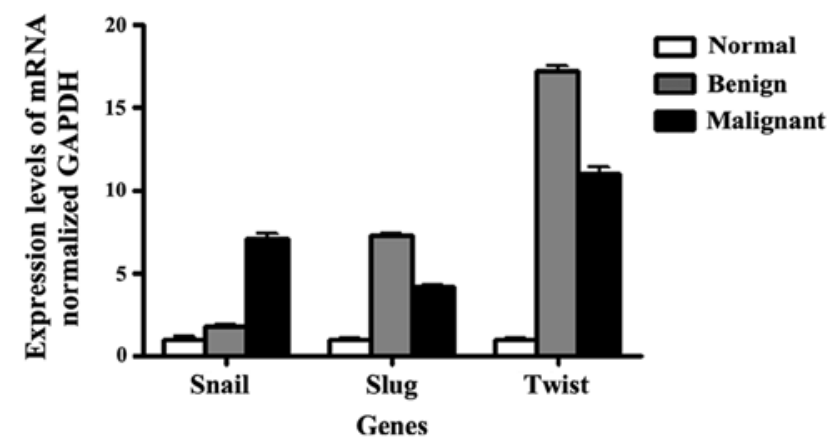

Figure 3. Quantification of EMT related transcription factors in clinical ovarian cancer samples. Total RNA was prepared using RNA extraction solution in human ovarian tissues obtained from patients and cDNA was synthesized by reverse transcription of MMLV reverse transcriptase. PCR reaction was performed using $2 \mathrm{X}$ SYBR green, ROX dye, and each forward and reverse primer including Snail, Slug, and Twist gene. ${ }^{*} \mathrm{P}<0.05$ vs. normal ovaries.

focusing on genes previously shown to be directly or indirectly involved in the EMT process. EMT-associated factors, including Snail, Slug and Twist, were overexpressed in the benign and malignant ovarian tumors compared to normal ovaries (Fig. 3). Technical validation by real-time PCR using SYBR green confirmed the relative overexpression of Snail by 1.8 - and 7.1-fold, Slug by 7.3- and 4.1-fold, and Twist by 17.2- and 11.0-fold in benign and malignant ovary samples, respectively. These transcriptional factors were significantly upregulated in benign and malignant ovarian tumors relative to normal ovaries.
Histopathological analysis of human normal ovaries and ovarian tumors. To investigate the histological features of normal ovaries or ovarian cancers, tissue slides were stained with H\&E. As shown in Fig. 4A, the specimen is a normal ovary specimen with a cystic follicle. A high magnification view of the cyst lining demonstrates the luteinized granulosa and theca cell layers on the top and bottom, respectively. Benign tumor contained mature cystic teratoma composed of multilocular cysts (Fig. 4B). Borderline tumors (mucinous endocervical type) consisted of gray white cystic masses with fragments of solid mass containing mucinous material and a capsule that had previously ruptured. Additionally, these tumors had a smooth inner lining wall that was thick and fibrotic. Low magnification revealed a papillary architecture indistinguishable from that seen in serous borderline tumors (Fig. 4C). Finally, serous adenocarcinoma was used to represent malignant tumors. The cut surface of these ovaries was yellow-gray and friable with extensive necrosis (Fig. 4D).

E-cadherin and vimentin expression in clinical tissue samples. To observe the expression of EMT marker in clinical samples, we conducted IHC analysis in normal ovaries and ovarian cancer tissues. Initially, vimentin was significantly upregulated in normal ovaries and malignant ovarian cancers (Fig. 5A and D). However, benign ovarian tumors showed low expression of the vimentin protein in the tissues (Fig. 5B and C). Epithelial cell marker E-cadherin immunoreactivity was increased in benign and malignant ovarian tumors relative to normal ovary tissues. Conversely, the protein was detected in $50 \%$ of borderline ovarian tumor samples. 

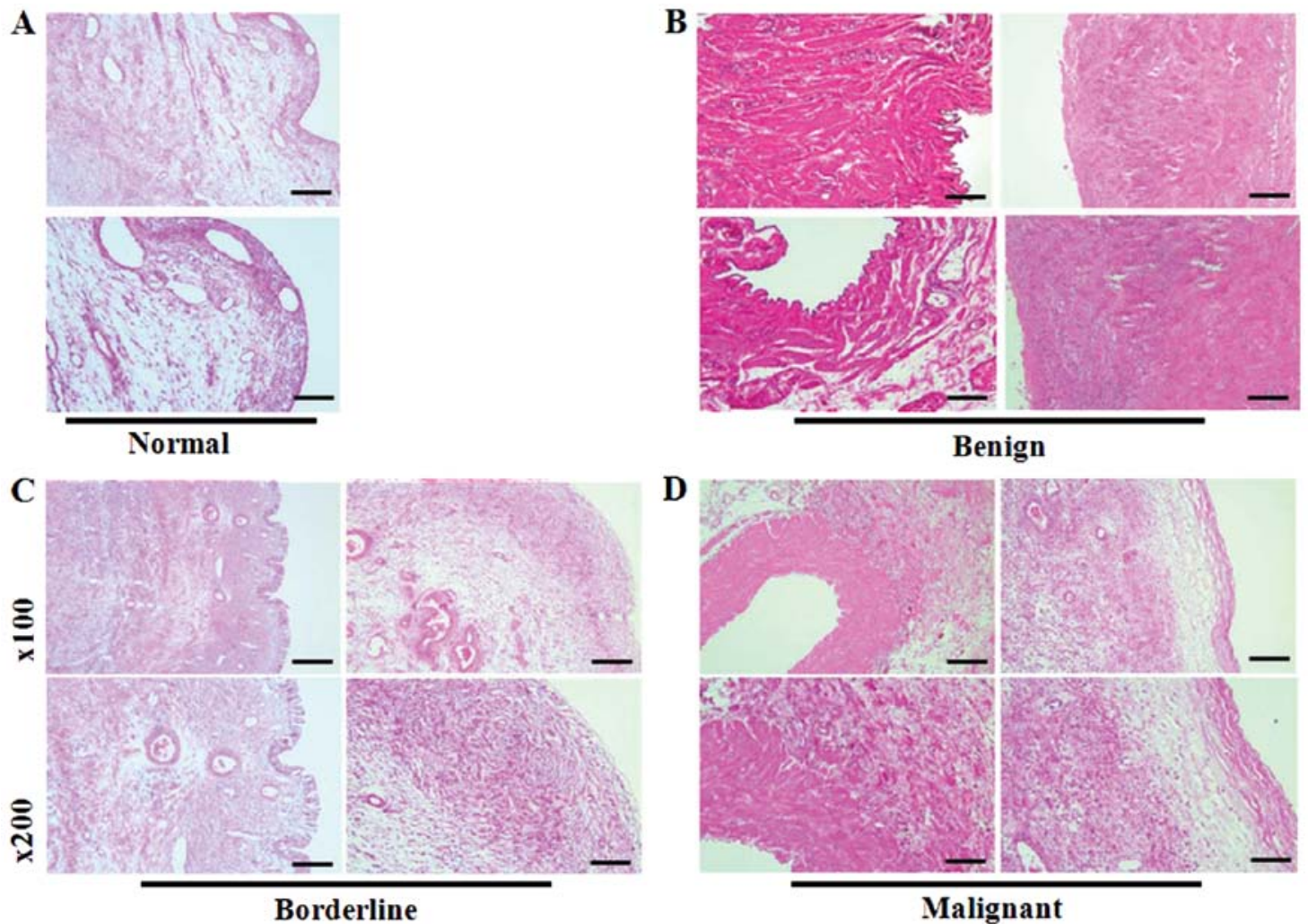

Figure 4. H\&E staining to confirm the histopathological analysis of human normal ovaries and ovarian cancers. Tissues were fixed in $4 \%$ normal formalin before histopathological analysis, then subjected to processing including hydration by ethanol and xylene, embedding by paraffin, and cutting with a microtome $(3 \mu \mathrm{m})$. After sufficient drying of tissues slides, samples were stained with hematoxylin and eosin. (A) Normal ovaries. (B) Benign. (C) Borderline. (D) Malignant. Magnification, x100 or x200.

\section{Discussion}

EMT is a phenomenon typically associated with epithelial cells in which the normal physiological state is characterized by abundant cortical E-cadherin expression and lack of vimentin. In contrast, normal ovarian surface epithelium is mesodermally-derived and expresses high levels of vimentin without cortical E-cadherin (25). The cellular features of EMT are a loss of epithelium-like polygonal morphology, apicobasolateral cell polarity and adhesive contacts, development of a fibroblast-like shape, reorganization of cytoskeletal filaments, increased cell motility, and induction of proteases for extracellular matrix (ECM) degradation, which is prerequisite for migration and invasion (26).

In this study, we confirmed the expression of EMT-related markers in three ovarian cancer cell lines, BG-1, OVCAR-3, and SKOV-3. The morphology of SKOV-3 cells revealed a fibroblastic shape that appeared to be mesenchymal. Additionally, vimentin protein was expressed in SKOV-3 ovarian cancer cells, but E-cadherin protein was not. The other two ovarian cancer cells showed features of epithelial cell type such as expression of E-cadherin and lack of vimentin protein. Based on these results, the expression of typical EMT-related markers, E-cadherin and vimentin, were altered based on ovarian cancer types, or morphology. Upon further investigation, alteration of EMT markers was observed in human clinical ovarian cancers or normal ovaries provided by patients. In the case of E-cadherin protein, benign ovarian cancers were upregulated by 3.4-fold when compared to normal ovaries or malignant ovarian cancers. Conversely, expression of vimentin protein was higher in normal ovaries or malignant ovarian cancer (1.5-fold). Expression of E-cadherin and vimentin protein was demonstrated by IHC staining in the human clinical samples. Generally, vimentin protein was strongly expressed in normal ovaries or malignant ovarian cancers, but E-cadherin protein was barely expressed in the samples. Based on these results, normal ovaries or malignant ovarian cancers displayed features such as mesenchymal cells. While most epithelia expressed abundant E-cadherin, it was absent from mesenchymally derived normal ovarian surface epithelia, and aberrant epithelial differentiation is an early event in epithelial ovarian carcinogenesis (27). In contrast to most carcinomas that lose E-cadherin expression with progression, its protein is abundant in primary differentiated ovarian carcinoma and available data indicate a subsequent decrease of E-cadherin staining in peritoneal metastases (28). Post-translational modification of E-cadherin function was suggested by data showing soluble E-cadherin in ascite or cystic fluids from ovarian cancer patients and ratios of cystic fluid/peripheral blood levels of soluble E-cadherin were significantly higher in cystadenocarcinoma and borderline ovarian tumors than benign tumors (29). Moreover, low E-cadherin expression was observed in more invasive tumors than benign tumors and associated with high tumor grade, presence of peritoneal seeding and low overall survival (30). Such a phenomenon seems paradoxical, but is re-expressed in metastatic lesions (31). The expression of E-cadherin is altered drastically during peritoneal dissemination in ovarian 


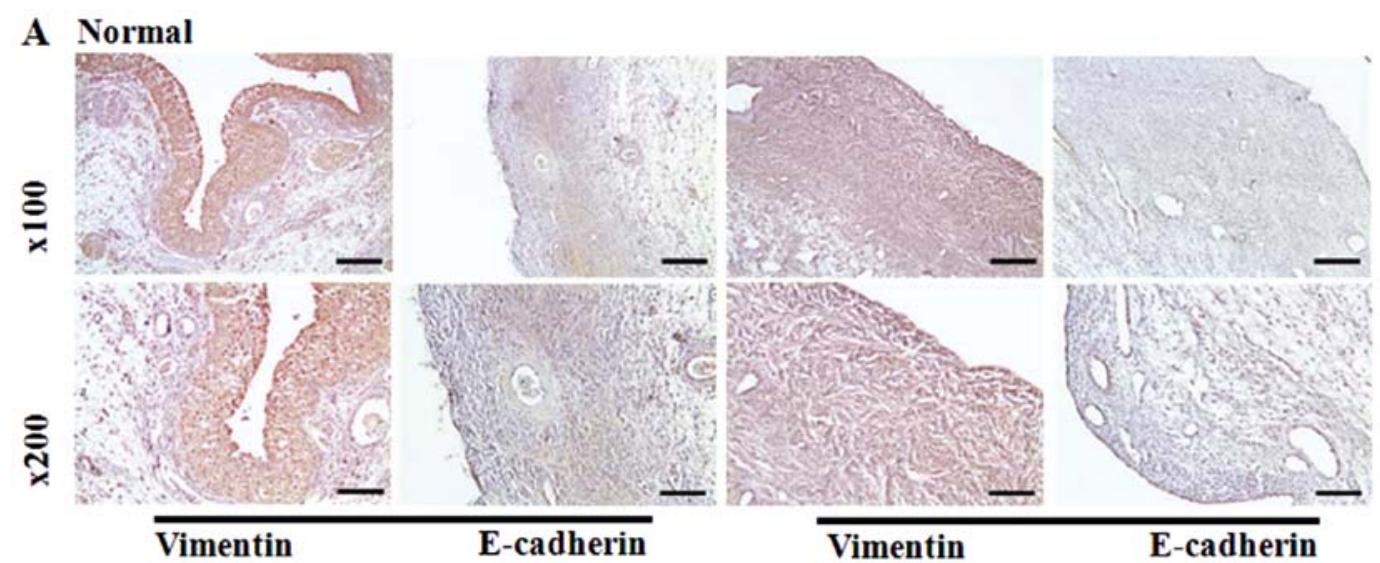

B Benign

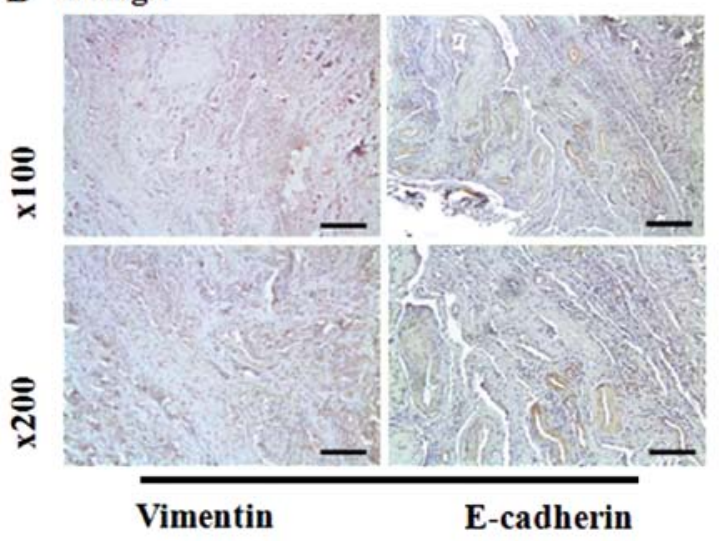

\section{Borderline}

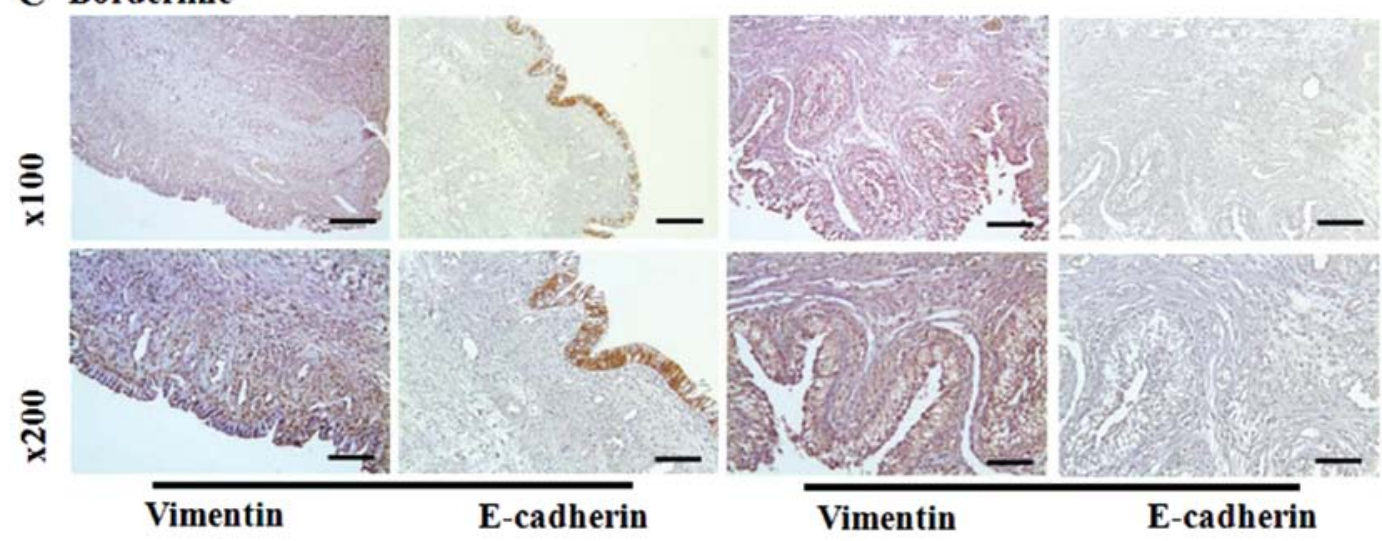

\section{Malignant}

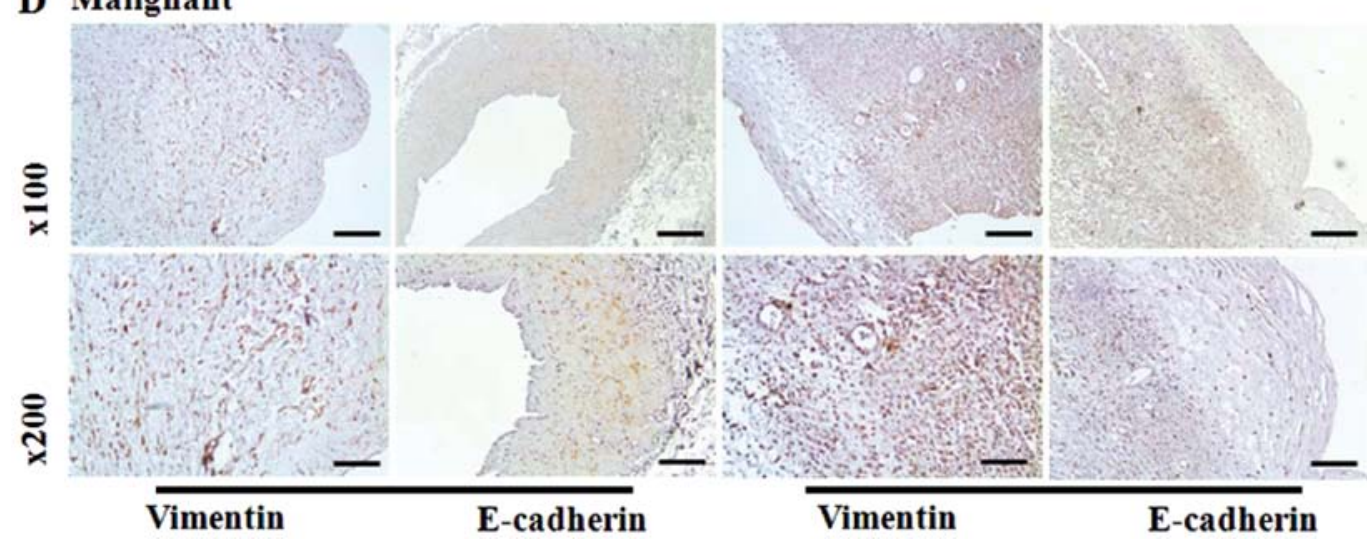

Figure 5. Localization of E-cadherin and vimentin proteins in clinical ovarian specimen. Tissue slides were incubated with $10 \%$ normal goat serum for $1 \mathrm{~h}$ and then treated with primary antibody, anti-E-cadherin (1:100 dilution) and anti-vimentin (1:100 dilution) at overnight. To confirm the protein expression, slides were treated with biotinylated anti-mouse secondary antibody (1:500 dilution), ABC kit reagent, and DAB substrate. (A) Normal ovaries. (B) Benign. (C) Borderline. (D) Malignant. Magnification, x100 or x200. 
cancer, and the specific repressors may play an important role in E-cadherin expression depending on the microenvironment of metastatic sites.

Ovarian carcinoma cells often undergo an EMT process before they detach or undergo metastasis. In ovarian carcinoma, the E-cadherin expression of cancer cells floating in ascites and at metastatic sites is lower than in primary ovarian tumors (32). Loss of E-cadherin gene expression is primarily due to upregulation of the transcriptional repressors, Snail, Slug, Twist or Sip1/ZEB1/2, which repress E-cadherin transcription (33). During malignant transformation, ovarian cancer cells lose E-cadherin-mediated cell-cell interaction, upregulate $\mathrm{N}$ - or P-cadherins and allow mesenchymal signaling through clustering of collagen binding integrins (e.g., $\alpha 2 \beta 1$ - and $\alpha 3 \beta 1$ integrin) (34). In this study, several transcription factors were analyzed to demonstrate the effect of altered expression of EMT-related marker by real-time PCR. This study showed the expression of transcriptional repressors for E-cadherin in normal ovaries and ovarian cancers. Among the repressors Snail, Slug, and Twist, malignant ovarian tumor expressed higher levels of Snail, but not Slug or Twist. The expression of Snail showed a stepwise increase from benign to malignant tumors. Snail disrupts the functions of adherens junctions as well as other cell-cell junctions, including tight junctions, and blocks E-cadherin transcription by binding to CANNTG (35). In another study, overexpression of Snail was shown to be correlated with a higher state or to be a marker of poor prognosis in malignancies because it confers resistance to apoptosis induced by DNA damage $(36,37)$. In ovarian cancer cell lines, upregulation of Snail and Slug has been correlated with resistance to radiation and paclitaxel and shown to participate directly in p53-mediated pro-survival signaling (38). Twist has been found to be expressed at high levels in a number of human cancers, including those of the breast, prostate, esophagus, and uterus, and is associated with EMT and intravasation (39). Although increased Slug and Twist gene expression was observed in this study, there was almost no alteration of EMT-related markers or transcriptional factors according to ovarian cancer types.

Taken together, in contrast to other epithelial cancers, E-cadherin protein was highly expressed in ovarian benign tumors, but barely expressed in normal ovaries. Additionally, the results indicate that Snail expression might be more important than that of other transcriptional repressors in ovarian tumor prognosis. Moreover, upregulation of Snail expression followed by the downregulation of E-cadherin enhances the invasiveness of ovarian tumors. Further studies may be needed to clarify the role of Snail in the aggressive behavior of ovarian cancer and the role of metastasis.

\section{Acknowledgements}

This study was supported by a grant from the Next-Generation BioGreen 21 Program (no. PJ009599), Rural Development Administration, Republic of Korea.

\section{References}

1. Lee JM, Dedhar S, Kalluri R and Thompson EW: The epithelialmesenchymal transition: new insights in signaling, development, and disease. J Cell Biol 172: 973-981, 2006.
2. Hay ED: The mesenchymal cell, its role in the embryo, and the remarkable signaling mechanisms that create it. Dev Dyn 233: 706-720, 2005 .

3. Larue L and Bellacosa A: Epithelial-mesenchymal transition in development and cancer: role of phosphatidylinositol 3' kinase/ AKT pathways. Oncogene 24: 7443-7454, 2005.

4. Thiery JP: Epithelial-mesenchymal transitions in tumour progression. Nat Rev Cancer 2: 442-454, 2002.

5. Thiery JP and Sleeman JP: Complex networks orchestrate epithelial-mesenchymal transitions. Nat Rev Mol Cell Biol 7: 131-142, 2006.

6. Vares G, Cui X, Wang B, Nakajima T and Nenoi M: Generation of breast cancer stem cells by steroid hormones in irradiated human mammary cell lines. PLoS One 8: e77124, 2008.

7. Thiery JP: Epithelial-mesenchymal transitions in development and pathologies. Curr Opin Cell Biol 15: 740-746, 2003.

8. Aktas B, Tewes M, Fehm T, Hauch S, Kimmig R and KasimirBauer S: Stem cell and epithelial-mesenchymal transition markers are frequently overexpressed in circulating tumor cells of metastatic breast cancer patients. Breast Cancer Res 11: R46, 2009.

9. Kurrey NK, Jalgaonkar SP, Joglekar AV, et al: Snail and slug mediate radioresistance and chemoresistance by antagonizing p53-mediated apoptosis and acquiring a stem-like phenotype in ovarian cancer cells. Stem Cells 27: 2059-2068, 2009.

10. Turley EA, Veiseh M, Radisky DC and Bissell MJ: Mechanisms of disease: epithelial-mesenchymal transition - does cellular plasticity fuel neoplastic progression? Nat Clin Pract Oncol 5: 280-290, 2008.

11. Sarrio D, Rodriguez-Pinilla SM, Hardisson D, Cano A, MorenoBueno G and Palacios J: Epithelial-mesenchymal transition in breast cancer relates to the basal-like phenotype. Cancer Res 68 : 989-997, 2008

12. Hemavathy K, Ashraf SI and Ip YT: Snail/slug family of repressors: slowly going into the fast lane of development and cancer. Gene 257: 1-12, 2000.

13. Ohkubo $\mathrm{T}$ and Ozawa M: The transcription factor Snail downregulates the tight junction components independently of E-cadherin downregulation. J Cell Sci 117: 1675-1685, 2004.

14. Savagner P, Kusewitt DF, Carver EA, et al: Developmental transcription factor slug is required for effective re-epithelialization by adult keratinocytes. J Cell Physiol 202: 858-866, 2005.

15. Nakayama $\mathrm{K}$, Nakayama $\mathrm{N}$, Katagiri $\mathrm{H}$ and Miyazaki $\mathrm{K}$ : Mechanisms of ovarian cancer metastasis: biochemical pathways. Int J Mol Sci 13: 11705-11717, 2012.

16. Batlle E, Sancho E, Franci C, et al: The transcription factor snail is a repressor of $\mathrm{E}$-cadherin gene expression in epithelial tumour cells. Nat Cell Biol 2: 84-89, 2000.

17. Grille SJ, Bellacosa A, Upson J, et al: The protein kinase Akt induces epithelial mesenchymal transition and promotes enhanced motility and invasiveness of squamous cell carcinoma lines. Cancer Res 63: 2172-2178, 2003.

18. Auersperg N, Wong AS, Choi KC, Kang SK and Leung PC: Ovarian surface epithelium: biology, endocrinology, and pathology. Endocr Rev 22: 255-288, 2001.

19. Hipp S, Berg D, Ergin B, et al: Interaction of Snail and p38 mitogen-activated protein kinase results in shorter overall survival of ovarian cancer patients. Virchows Arch 457: 705-713, 2010.

20. Okamoto S, Okamoto A, Nikaido T, et al: Mesenchymal to epithelial transition in the human ovarian surface epithelium focusing on inclusion cysts. Oncol Rep 21: 1209-1214, 2009.

21. Wong AS and Auersperg N: Normal ovarian surface epithelium. Cancer Treat Res 107: 161-183, 2002.

22. Strauss R, Li ZY, Liu Y, et al: Analysis of epithelial and mesenchymal markers in ovarian cancer reveals phenotypic heterogeneity and plasticity. PLoS One 6: e16186, 2011.

23. Cho EY, Choi Y, Chae SW, Sohn JH and Ahn GH: Immunohistochemical study of the expression of adhesion molecules in ovarian serous neoplasms. Pathol Int 56: 62-70, 2006.

24. Ahmed N, Maines-Bandiera S, Quinn MA, Unger WG, Dedhar S and Auersperg N: Molecular pathways regulating EGF-induced epithelio-mesenchymal transition in human ovarian surface epithelium. Am J Physiol Cell Physiol 290: C1532-C1542, 2006.

25. Ahmed N, Thompson EW and Quinn MA: Epithelialmesenchymal interconversions in normal ovarian surface epithelium and ovarian carcinomas: an exception to the norm. J Cell Physiol 213: 581-588, 2007. 
26. Demir AY, Groothuis PG, Nap AW, et al: Menstrual effluent induces epithelial-mesenchymal transitions in mesothelial cells. Hum Reprod 19: 21-29, 2004.

27. Symowicz J, Adley BP, Gleason KJ, et al: Engagement of collagen-binding integrins promotes matrix metalloproteinase-9-dependent E-cadherin ectodomain shedding in ovarian carcinoma cells. Cancer Res 67: 2030-2039, 2007.

28. Imai T, Horiuchi A, Shiozawa T, et al: Elevated expression of E-cadherin and alpha-, beta-, and gamma-catenins in metastatic lesions compared with primary epithelial ovarian carcinomas. Hum Pathol 35: 1469-1476, 2004.

29. Sundfeldt K, Ivarsson K, Rask K, Haeger M, Hedin L and Brannstrom M: Higher levels of soluble E-cadherin in cyst fluid from malignant ovarian tumours than in benign cysts. Anticancer Res 21: 65-70, 2001

30. Vergara D, Merlot B, Lucot JP, et al: Epithelial-mesenchymal transition in ovarian cancer. Cancer Lett 291: 59-66, 2010.

31. Liu J, Ikeguchi M, Nakamura S and Kaibara N: Re-expression of the cadherin-catenin complex in lymph nodes with metastasis in advanced gastric cancer: the relationship with patient survival. J Exp Clin Cancer Res 21: 65-71, 2002.

32. Lengyel E: Ovarian cancer development and metastasis. Am J Pathol 177: 1053-1064, 2010.

33. Elloul S, Elstrand MB, Nesland JM, et al: Snail, Slug, and Smad-interacting protein 1 as novel parameters of disease aggressiveness in metastatic ovarian and breast carcinoma. Cancer 103: 1631-1643, 2005.
34. Patel IS, Madan P, Getsios S, Bertrand MA and MacCalman CD: Cadherin switching in ovarian cancer progression. Int J Cancer 106: 172-177, 2003.

35. Cano A, Perez-Moreno MA, Rodrigo I, et al: The transcription factor snail controls epithelial-mesenchymal transitions by repressing E-cadherin expression. Nat Cell Biol 2: 76-83, 2000.

36. Yin T, Wang C, Liu T, Zhao G, Zha Y and Yang M: Expression of snail in pancreatic cancer promotes metastasis and chemoresistance. J Surg Res 141: 196-203, 2007.

37. Miow QH, Tan TZ, Ye J, et al: Epithelial-mesenchymal status renders differential responses to cisplatin in ovarian cancer. Oncogene: May 26, 2014 (Epub ahead of print) doi: 10.1038/ onc.2014.136.

38. Haslehurst AM, Koti M, Dharsee M, et al: EMT transcription factors snail and slug directly contribute to cisplatin resistance in ovarian cancer. BMC Cancer 12: 91, 2012.

39. Lo HW, Hsu SC, Xia W, et al: Epidermal growth factor receptor cooperates with signal transducer and activator of transcription 3 to induce epithelial-mesenchymal transition in cancer cells via up-regulation of TWIST gene expression. Cancer Res 67: 9066-9076, 2007. 